\title{
Could physical exercise be an effective treatment for adults with borderline personality disorder?
}

Dear Editor,

After an exhaustive search of the literature, we were astonished to discover no previous research study investigating the therapeutic effects of physical exercise for adults with borderline personality disorder (BPD). Accordingly, we would like to briefly present a rationale for studying the potential benefits of physical exercise for adults with BPD.

BPD is characterized by an instability in self-image, relationships, and emotions (Gunderson et al., 2018). Of these symptoms, emotion dysregulation is among the most commonly targeted symptoms in psychotherapy because it can impact the therapeutic relationship and be associated with a lower quality of life (Gunderson et al., 2018). BPD is highly comorbid with other psychiatric disorders, especially anxiety and depressive disorders (Gunderson et al., 2018), and physical illnesses, most commonly cardiovascular diseases, metabolic syndrome, and diabetes (Doering, 2019). In fact, cardiovascular diseases, along with respiratory and endocrine diseases, account for $32 \%$ of deaths among people with cluster B personality disorders which include BPD (Cailhol et al., 2017). However, no study to our knowledge has yet examined the effects of physical exercise in adults with BPD.

Physical exercise is an effective intervention to reduce emotion dysregulation and to increase positive affects in healthy adults in an ecological context (Bernstein et al., 2019). More active individuals have reported a quicker return to a neutral or positive affective state after feeling negative affects. Moreover, a single session of physical exercise is effective at increasing positive affects in a short-term in adults with anxiety or depressive disorders (Meyer et al., 2016).

In adults with BPD, emotion dysregulation has been linked to a dysfunction in prefrontal cortex and amygdala among other neurobiological structures (Ruocco and Carcone, 2016). Acute effect of physical exercise on those structures has been studied and shows promising results for the emotion regulation in adults with BPD (Schneider et al., 2009).

Chronic physical exercise is also an evidence-based intervention to reduce symptom severity in adults with major depressive disorder, generalized anxiety disorder (Ravindran et al., 2016) and schizophrenia (Dauwan et al., 2016). A recent meta-analysis concluded that physical exercise intervention is an efficient way to increase cardiovascular fitness and reduce cardiovascular disease risk in adults with severe mental illness (Vancampfort et al., 2017).

Therefore, physical exercise could be tested as an adjunct treatment with adults with BPD to improve emotion regulation, treat comorbid psychiatric symptoms, and reduce cardiovascular disease risk. Future studies need to: i) address the safety of physical intervention in adults with BPD; ii) identify the exercise modalities associated with a good adherence rate, such as preferences and barriers in this population; and iii) evaluate the short- and long-term effects of physical exercise on BPD, its symptoms and its comorbidities.

Declaration of Competing Interest

None

St-Amour $\mathrm{S}^{\mathrm{a}, \mathrm{b} *}$, Cailhol L ${ }^{\mathrm{b}}$, Ruocco AC ${ }^{\mathrm{c}}$, Bernard $\mathrm{P}^{\mathrm{a}, \mathrm{b}}$

${ }^{*}$ Corresponding author: stamour.samuel@gmail.com

a Université du Québec à Montréal, Physical activity sciences department, Montreal, Quebec, Canada

${ }^{\mathrm{b}}$ Mental health University Institute of Montreal Research Center, Montreal, Quebec, Canada

${ }^{c}$ University of Toronto, Department of Psychology (Scarborough), Toronto, Ontario, Canada 


\section{References}

Bernstein, E.E., Curtiss, J.E., Wu, G.W.Y., Barreira, P.J., McNally, R.J., 2019. Exercise and emotion dynamics: An experience sampling study. Emot. Wash. DC 19, 637-644. https://doi.org/10.1037/emo0000462

Cailhol, L., Pelletier, É., Rochette, L., Laporte, L., David, P., Villeneuve, É., Paris, J., Lesage, A., 2017. Prevalence, Mortality, and Health Care Use among Patients with Cluster B Personality Disorders Clinically Diagnosed in Quebec: A Provincial Cohort Study, 2001-2012. Can. J. Psychiatry Rev. Can. Psychiatr. 62, 336-342. https://doi.org/10.1177/0706743717700818

Dauwan, M., Begemann, M.J.H., Heringa, S.M., Sommer, I.E., 2016. Exercise Improves Clinical Symptoms, Quality of Life, Global Functioning, and Depression in Schizophrenia: A Systematic Review and Meta-analysis. Schizophr. Bull. 42, 588-599. https://doi.org/10.1093/schbul/sbv164

Doering, S., 2019. Borderline Personality Disorder in Patients With Medical Illness: A Review of Assessment, Prevalence, and Treatment Options. Psychosom. Med. 81, 584-594. https://doi.org/10.1097/PSY.0000000000000724

Gunderson, J.G., Herpertz, S.C., Skodol, A.E., Torgersen, S., Zanarini, M.C., 2018. Borderline personality disorder. Nat. Rev. Dis. Primer 4, 18029. https://doi.org/10.1038/nrdp.2018.29

Meyer, J.D., Koltyn, K.F., Stegner, A.J., Kim, J.-S., Cook, D.B., 2016. Influence of Exercise Intensity for Improving Depressed Mood in Depression: A Dose-Response Study. Behav. Ther. 47, 527537. https://doi.org/10.1016/j.beth.2016.04.003

Ruocco, A.C., Carcone, D., 2016. A Neurobiological Model of Borderline Personality Disorder: Systematic and Integrative Review. Harv. Rev. Psychiatry 24, 311-329. https://doi.org/10.1097/ HRP.0000000000000123

Schneider, M., Graham, D., Grant, A., King, P., Cooper, D., 2009. Regional brain activation and affective response to physical activity among healthy adolescents. Biol. Psychol. 82, 246-252. https://doi.org/10.1016/j.biopsycho.2009.08.003

Vancampfort, D., Rosenbaum, S., Schuch, F., Ward, P.B., Richards, J., Mugisha, J., Probst, M., Stubbs, B., 2017. Cardiorespiratory Fitness in Severe Mental IIIness: A Systematic Review and Meta-analysis. Sports Med. 47, 343-352. https://doi.org/10.1007/s40279-016-0574-1 